\title{
CHRONOLOGICAL PRODUCTS, ENERGY-MOMENTUM TENSORS OF SCALAR FIELDS FOR PARTICLE GENERATIONS, AND INDEFINITE METRICS
}

\author{
Yu.V. Kulish \\ Ukrainian State University of Railway Transport \\ Sq. Feuerbach 7, Kharkiv region, 61050, Ukraine \\ e-mail: Yu.V.Kulish@gmail.com \\ Received February 22, 2018
}

The solutions of generalized Klein-Gordon equations are considered. The generalizations of the Klein-Gordon equation allow one to derive convergent integrals for the Green functions of these equations. The generalized equations are presented as products of the operators for the Klein-Gordon equation with different masses. The solutions of derived homogeneous equations (total fields) are sums of fields corresponding to particles with the same values of the spin, the electric charge, the parities, but with different masses. Such particles are grouped into the kinds (families, dynasties) with members which are the generations. The chronological products of the scalar fields for kinds of particles are obtained at arbitrary quantity of the generations. These chronological products are the causal Green functions of generalized Klein-Gordon equations. The Lagrangians for the generalized Klein-Gordon equations of arbitrary order are derived. These Lagrangians are used to obtain the energy-momentum tensors for the particle kinds at arbitrary quantity of generations. It is shown that the generalized Hamiltonians (for total fields) have got positive eigenvalues for all the generations. These results are derived with the use of the indefinite metrics.

KEY WORDS: generalized Klein-Gordon equations, generations of particles, chronological products of fields, Green functions, Lagrangians, particle energies, particle moments, indefinite metrics.

\section{ХРОНОЛОГІЧНІ ДОБУТКИ, ТЕНЗОРИ ЕНЕРГІЇ-ІМПУЛЬСУ СКАЛЯРНИХ ПОЛІВ ДЛЯ ПОКОЛІНЬ ЧАСТИНОК ТА ІНДЕФІНІТНА МЕТРИКА}

Ю.В. Куліш

Український державний університет залізничного транспорту

м. Фейєрбаха 7, Харків, 61050, Украӥна

Розглянуто розв'язки узагальнених рівнянь Клейна-Гордона. Узагальнення рівняння Клейна-Гордона дозволяє одержати збіжні інтеграли для функцій Гріна цих рівнянь. Узагальнені рівняння представляють собою добутки операторів рівняння Клейна-Гордона 3 різними масами. Розв'язки одержаних однорідних рівнянь (повні поля) є суми полів відповідних частинкам 3 однаковими значеннями спіну, електричного заряду, парностей, але 3 різними масами. Такі частинки групуються в роди (сім’і, династії) з членами, які є поколіннями. Одержано хронологічні добутки скалярних полів для родів частинок з довільною кількістю поколінь. Ці хронологічні добутки є причинні функції Гріна узагальнених рівнянь КлейнаГордона. Одержано лагранжияни для узагальнених рівнянь Клейна-Гордона довільного порядку. Ці лагранжіяни використано для знаходження тензорів енергії-імпульсу для родів частинок з довільною кількістю поколінь. Показано, що узагальнені гамільтоніяни (для повних полів) мають додатні власні значення для всіх поколінь. Ці результати одержано 3 використанням індефінітної метрики.

КЛЮЧОВІ СЛОВА: Узагальнені рівняння Клейна-Гордона, покоління частинок, хронологічні добутки полів, функції Гріна, лагранжіяни, енергії частинок, імпульси частинок, індефінітна метрика.

\section{ХРОНОЛОГИЧЕСКИЕ ПРОИЗВЕДЕНИЯ, ТЕНЗОРЫ ЭНЕРГИИ-ИМПУЛЬСА СКАЛЯРНЫХ ПОЛЕЙ ДЛЯ ПОКОЛЕНИЙ ЧАСТИЦ И ИНДЕФИНИТНАЯ МЕТРИКА Ю.В. Кулиш}

Украинский государственный университет железнодорожного транспорта пл. Фейербаха 7, Харьков, 61050, Украина

Рассмотрены решения обобщенных уравнений Клейна-Гордона. Обобщение уравнений Клейна-Гордона позволяет получить сходящиеся интегралы для функций Грина этих уравнений. Обобщенные уравнения представляют собой произведения операторов уравнения Клейна-Гордона с разными массами. Решения полученных однородных уравнений (полные поля) являются суммами полей, соответствующих частицам с одинаковыми величинами спина, электрического заряда, четностей, но с разными массами. Такие частицы группируются в рода (семьи, династии), которые являются поколениями. Получены хронологические произведения скалярных полей для родов частиц с произвольным количеством поколений. Эти хронологические произведения являются причинными функциями Грина обобщенных уравнений Клейна-Гордона. Получены лагранжианы обобщенных уравнений Клейна-Гордона произвольного порядка. Эти лагранжианы использованы для получения тензоров энергии-импульса родов частиц с произвольным количеством поколений. Показано, что обобщенные гамильтонианы (для полных полей) имеют положительные собственные числа для всех поколений. Эти результаты получены при использовании индефинитной метрики

КЛЮЧЕВЫЕ СЛОВА: обобщенные уравнения Клейна-Гордона, поколения частиц, хронологические произведения полей, функцуии Грина, лагранжианы, энергии частиц, импульсы частиц, индефинитная метрика.

It is known that reaction amplitudes are calculated with the use of the particle propagators and the vertex functions [1-8]. In the momentum representation all the particle propagators can be presented as products of the propagator for the 
spinless particle and corresponding projection operator. The particle propagator of the spinless particle in coordinate representation is the causal Green function of the Klein-Gordon equation (the Klein-Gordon-Fock equation). It means that the Green functions of the Klein-Gordon equation and other wave equations for any spin particles are infinite improper four-fold integrals with respect to momentum variables. In Refs. [9, 10] it is shown that corresponding fourfold integrals for the Klein-Gordon and Dirac equations, as well as triple integral for the Yukawa potential, diverge. To eliminate these divergences the integrals for the Green functions have been generalized in Refs. [9, 10]. New Green functions correspond to equations with higher partial derivatives. Among these equations the generalized Klein-Gordon equation is very important, as many features of generalized equations may be seen. The $\Phi(x)$ solutions of the generalized Klein-Gordon equations can be presented as sum of solutions of the Klein-Gordon equations for different particle masses. These sums correspond to some sets of particles. Therefore, values related to the solutions of the generalized Klein-Gordon equations can have some feature in a comparison with similar values for the Klein-Gordon equations. In particular, it is of importance to study Lagrangians and energy-momentum tensors for generalized equations.

The aim of present paper is the study of some topics related to the solutions of the generalized Klein-Gordon equations. Similar topics for one field are well known [1-8]. So, it is shown that the chronological product of quantized free solutions for the generalized Klein-Gordon equation is the causal Green function of this equation. In terms of the total fields the Lagrangians, the energy-momentum tensors, and a generalized Hamiltonian are derived. In particular, the question on the positive determination of the generalized Hamiltonian is investigated. In addition, conditions, which permit one to derive some general relations for the solution of the Klein-Gordon equations, are studied.

\section{RELATION OF CHRONOLOGICAL PRODUCTS FOR FIELDS AND GREEN FUNCTIONS}

It is known that the $\delta\left(x-x^{\prime}\right)$ - function and the $G\left(x-x^{\prime}\right)$ Green functions of differential equation can be expanded in the set of eigenfunctions for the operator of this differential equation [11]. Accordingly to Refs. [2-6] the vacuum average of the chronological product for the scalar fields is related to the $D(x, m)_{c}$ causal Green function. This relation is given by

$$
\left\langle 0\left|T\left(\varphi(x), \varphi(y)^{+}\right)\right| 0\right\rangle=-i D(x-y, m)_{c}, \quad D(x, m)_{c}=\frac{1}{(2 \pi)^{4}} \int \frac{e^{-i q x}}{m^{2}-q^{2}-i \varepsilon} d^{4} q
$$

The generalized Klein-Gordon equations and their $\Phi(x)$ solutions (which are named as total fields and fields of particle generations) are considered in Appendix. It is of interest to study the relation between the chronological product of the quantized $\Phi(x)$ total fields, which are the solutions of the homogeneous equation (A.1), and the Green function of this equation.

For the chronological product of the $A(x)$ operator and the $B(y)$ operator usual definition can be exploited:

$$
T(A(x) B(y))=\theta\left(x_{0}-y_{0}\right) A(x) \cdot B(y)+\theta\left(y_{0}-x_{0}\right) B(y) \cdot A(x)
$$

The discontinuous function $\theta\left(x_{0}\right)$ can be presented by the improper integral

$$
\theta\left(x_{0}\right)=\frac{1}{2 \pi i} \int_{-\infty}^{\infty} \frac{e^{i q x_{0}} d q}{q-i \varepsilon}
$$

We propose that the total $\Phi(x)$ quantized field is given by

$$
\begin{aligned}
& \Phi(x)=\frac{1}{\sqrt{(2 \pi)^{3}}} \sum_{k=1}^{N} \sqrt{A_{k}} e^{i \delta_{k}} \int \frac{d^{3} p}{2 \omega_{k}}\left[e^{-i p x} a(\vec{p})_{k}+e^{i p x} b(\vec{p})_{k}^{+}\right]=\sum_{k=1}^{N} \sqrt{A_{k}} e^{i \delta_{k}} \cdot \varphi(x)_{k}, \\
& \varphi(x)_{k}=\frac{1}{\sqrt{(2 \pi)^{3}}} \int \frac{d^{3} p}{2 \omega_{k}}\left[e^{-i p x} a(\vec{p})_{k}+e^{i p x} b(\vec{p})_{k}^{+}\right], \\
& \omega_{k}=p_{0 k}=\sqrt{\vec{p}^{2}+m_{k}^{2}}
\end{aligned}
$$


where $\delta_{k}$ are some phases. The $\delta_{k}$ phases can give parameters of mixing for composite states. The $\varphi(x)_{k}$ field is the scalar quantized free field of the $k$ number generation.

We use the indefinite metrics. The covariant normalization condition, the non-zero commutators of the $a(\vec{p})_{k}$ $\left(b(\vec{p})_{k}\right)$ annihilation operators for particles (antiparticles) and the $a(\vec{p})_{k}{ }^{+}\left(b(\vec{p})_{k}{ }^{+}\right)$creation operators are the generalizations of usual commutators for one particle:

$$
\begin{aligned}
& \left\langle\vec{p}_{1}, k_{1}, \sigma_{1} \mid \vec{p}_{2}, k_{2}, \sigma_{2}\right\rangle=2 \omega_{k_{1}}(-1)^{k_{1}+1} \delta_{k_{1} k_{2}} \delta_{\sigma_{1} \sigma_{2}} \delta\left(\vec{p}_{1}-\vec{p}_{2}\right), \\
& {\left[a\left(\vec{p}_{1}\right)_{k_{1}, \sigma_{1}}, a\left(\vec{p}_{2}\right)^{+}{ }_{k_{2}, \sigma_{2}}\right]=2 \omega_{k_{1}}(-1)^{k_{1}+1} \delta_{k_{1} k_{2}} \delta_{\sigma_{1} \sigma_{2}} \delta\left(\vec{p}_{1}-\vec{p}_{2}\right),} \\
& {\left[b\left(\vec{p}_{1}\right)_{k_{1}, \sigma_{1}}, b\left(\vec{p}_{2}\right)^{+}{ }_{k_{2}, \sigma_{2}}\right]=2 \omega_{k_{1}}(-1)^{k_{1}+1} \delta_{k_{1} k_{2}} \delta_{\sigma_{1} \sigma_{2}} \delta\left(\vec{p}_{1}-\vec{p}_{2}\right),} \\
& \langle 0 \mid 0\rangle=1
\end{aligned}
$$

where $\sigma_{1}, \sigma_{2}$ are some discrete quantum numbers. The signs in the relations (5) agree with the signs of the $A_{k}$ coefficients $\left(A_{k}=(-1)^{k+1}\left|A_{k}\right|\right)$.

Using the formulae (2) and (4) the chronological product of the total scalar fields may be written as

$$
\begin{aligned}
& T\left(\Phi(x) \Phi(y)^{+}\right)=\frac{1}{(2 \pi)^{3}} \sum_{k_{1}, k_{2}=1}^{N} \sqrt{A_{k_{1}}}\left(\sqrt{A_{k_{2}}}\right)^{*} \exp \left[i\left(\delta_{k_{1}}-\delta_{k_{2}}\right)\right] \times \\
& \times \int \frac{d^{3} p_{1} d^{3} p_{2}}{4 \omega_{k_{1}} \omega_{k_{2}}}\left\{\exp \left[i\left(-p_{1} x+p_{2} y\right)\right]\left\langle 0\left|a\left(\vec{p}_{1}\right)_{k_{1}} a\left(\vec{p}_{2}\right)_{k_{2}}^{+}\right| 0\right\rangle \theta\left(x_{0}-y_{0}\right)+\exp \left[i\left(p_{1} x-p_{2} y\right)\right]\left\langle 0\left|b\left(\vec{p}_{2}\right)_{k_{2}} b\left(\vec{p}_{1}\right)_{k_{1}}^{+}\right| 0\right\rangle \theta\left(y_{0}-x_{0}\right)\right\}
\end{aligned}
$$

The vacuum matrix elements of the products for the annihilation operators and the creation operators equal the corresponding commutators (5). After the substitutions of these commutators in the chronological product (6) we can write:

$$
T\left(\Phi(x) \Phi(y)^{+}\right)=\frac{1}{(2 \pi)^{3}} \sum_{k=1}^{N}\left|A_{k}\right|(-1)^{k+1} \int \frac{d^{3} p}{2 \omega_{k}}\left\{\exp [-i p(x-y)] \theta\left(x_{0}-y_{0}\right)+\exp [i p(x-y)] \theta\left(y_{0}-x_{0}\right)\right\}
$$

Now it can be used the representation (3) for discontinuous function. Then we obtain four-fold integral for (7)

$$
\begin{aligned}
& T\left(\Phi(x) \Phi(y)^{+}\right)=\frac{1}{(2 \pi)^{4} i} \sum_{k=1}^{N} A_{k} \int \frac{d^{3} p}{2 \omega_{k}} \int_{-\infty}^{\infty} \frac{d q}{q-i \varepsilon} . \\
& \cdot\left\{\exp \left[i\left(q-\omega_{k}\right)\left(x_{0}-y\right)_{0}\right] \exp [i \vec{p}(\vec{x}-\vec{y})]+\exp \left[-i\left(q-\omega_{k}\right)\left(x_{0}-y\right)_{0}\right] \exp [-i \vec{p}(\vec{x}-\vec{y})]\right\}
\end{aligned}
$$

In Ref. [12] has been shown that for $N \geq 3$

$$
\sum_{k=1}^{N} A_{k} \int \frac{d^{3} p}{2 \omega_{k}} \exp [i \vec{p}(\vec{x}-\vec{y})]=\sum_{k=1}^{N} A_{k} \int \frac{d^{3} p}{2 \omega_{k}} \exp [-i \vec{p}(\vec{x}-\vec{y})]
$$

Note that each term in (9) is diverging triple integral. We change the variable of integration $q$ in (8) by means of such variables: $q=\omega_{k}-p_{0}$ in the first term and $q=\omega_{k}+p_{0}$ in the second term. Then the T-product (8) with the use of (9) can be written as

$$
\begin{aligned}
& T\left(\Phi(x) \Phi(y)^{+}\right)=\frac{1}{(2 \pi)^{4} i} \sum_{k=1}^{N} A_{k} \int \frac{d^{4} p}{2 \omega_{k}} \exp \{-i p(x-y)\} \cdot\left[\frac{1}{\omega_{k}-p_{0}-i \varepsilon}+\frac{1}{\omega_{k}+p_{0}-i \varepsilon}\right]= \\
& =\frac{1}{(2 \pi)^{4} i} \sum_{k=1}^{N} A_{k} \int \frac{d^{4} p}{2 \omega_{k}} \exp [-i p(x-y)] \cdot \frac{2 \omega_{k}}{\omega_{k}^{2}-p_{o}^{2}-2 i \varepsilon \omega_{k}}
\end{aligned}
$$


The $\omega_{k}$ value (4) is positive and the $2 i \varepsilon \omega_{k}$-value is the imaginary positive infinitesimal numbers. Therefore, we may denote the $2 i \varepsilon \omega_{k}$ value in (10) as the $i \varepsilon$ value. Now the T-product of the total fields (10) is given by

$$
T\left(\Phi(x) \Phi(y)^{+}\right)=\frac{1}{(2 \pi)^{4} i} \sum_{k=1}^{N} A_{k} \int d^{4} p \frac{\exp [-i p(x-y)]}{m_{k}^{2}-p^{2}-i \varepsilon}=-i \sum_{k=1}^{N} A_{k} D\left(x-y, m_{k}\right)_{c}=-i \bar{G}(x-y)_{c}
$$

Thus, the chronological product of the total scalar fields (4) is related to the causal Green function (A.10) of the generalized Klein-Gordon equation (A.1), similarly to the (1) relation for one particle. This relation is the consequence of the indefinite metrics, expressed by the commutators (5), and the equality (9) at $N \geq 3$.

\section{Lagrangians for generalized Klein-Gordon equations}

In Ref. [13] the Lagrangians for the generalized Dirac equations are derived. Therefore it is of interest to obtain the Lagrangian for the generalized Klein-Gordon equations. Operators of the generalized Klein-Gordon equations (A.1) are polynomials with respect to the $\square$ operator of d'Alembert (d'Alembertian). They can be written as

$$
\prod_{n=1}^{N}\left(\square+m_{n}{ }^{2}\right)=\sum_{n=1}^{N} S\left(m_{1}^{2}, m_{2}^{2}, \cdots, m_{N}^{2}\right)_{N-n} \square^{n}
$$

The $S_{k}$ values are elementary symmetric functions [14]. They equal:

$$
\begin{aligned}
& S\left(m_{1}{ }^{2}, m_{2}{ }^{2}, \cdots, m_{N}{ }^{2}\right)_{0}=1, \\
& S\left(m_{1}^{2}, m_{2}^{2}, \cdots, m_{N}^{2}\right)_{1}=m_{1}^{2}+m_{2}^{2}+\cdots+m_{N}^{2}, \\
& S\left(m_{1}^{2}, m_{2}^{2}, \cdots, m_{N}^{2}\right)_{2}=m_{1}{ }^{2} m_{2}{ }^{2}+m_{1}{ }^{2} m_{3}^{2}+\cdots+m_{N-1}{ }^{2} m_{N}{ }^{2}, \\
& S\left(m_{1}{ }^{2}, m_{2}{ }^{2}, \cdots, m_{N}{ }^{2}\right)_{3}=m_{1}{ }^{2} m_{2}{ }^{2} m_{3}{ }^{2}+m_{1}{ }^{2} m_{2}{ }^{2} m_{4}{ }^{2}+\cdots+m_{N-2}{ }^{2} m_{N-1}{ }^{2} m_{N}{ }^{2}, \ldots, \\
& S\left(m_{1}^{2}, m_{2}^{2}, \cdots, m_{N}^{2}\right)_{N}=m_{1}^{2} m_{2}^{2} m_{3}^{2} \cdots m_{N-1}^{2} m_{N}^{2} \text {. }
\end{aligned}
$$

For these functions the formula can be written at $k>1$

$$
S\left(m_{1}^{2}, m_{2}^{2}, \cdots, m_{N}^{2}\right)_{k}=\sum_{i_{k}>i_{k-1}>\cdots>i_{2}>i_{1} \geq 1}^{N} m_{i_{1}}{ }^{2} m_{i_{2}}{ }^{2} m_{i_{3}}{ }^{2} \cdots m_{i_{k-1}}{ }^{2} m_{i_{k}}{ }^{2}
$$

In the case of equal masses $\left(m_{1}{ }^{2}=m_{2}{ }^{2}=\cdots=m_{N}{ }^{2}=m^{2}\right)$ the elementary symmetric functions are related to the binomial coefficients $C_{N}^{k}$

$$
S\left(m^{2}, m^{2}, \cdots, m_{N}^{2}\right)_{k}=m^{2 k} C_{N}^{k}=m^{2 k} \frac{N !}{k !(N-k) !}
$$

As the operators of generalized Klein-Gordon equations (A.1) include the partial derivatives of the $2 N$ order and they are polynomials, Lagrangians for these equations must have polynomial structure. Let us denote

$$
\begin{aligned}
& \Phi(x)_{\mu_{1} \mu_{2} \cdots \mu_{n}}=\partial_{\mu_{1}} \partial_{\mu_{2}} \cdots \partial_{\mu_{n}} \Phi(x)=\Phi x_{\mu}^{n}, \\
& \Phi(x)_{\mu_{1} \mu_{2} \cdots \mu_{n}}=\partial_{\mu_{1}} \partial_{\mu_{2}} \cdots \partial_{\mu_{n}} \Phi(x)^{+}=\Phi x_{\mu}^{+n} . \\
& \partial x_{\mu}{ }^{n}=\partial_{\mu_{1}} \partial_{\mu_{2}} \cdots \partial_{\mu_{n}}=\frac{\partial^{n}}{\partial x_{\mu_{1}} \partial x_{\mu_{2}} \cdots \partial x_{\mu_{n}}}
\end{aligned}
$$


In general, the Lagrangian can depend on the x coordinates, $\Phi(x), \Phi(x)^{+}$fields, and their derivatives (16) for $1 \leq n \leq N$. Using the least action principle (the Ostrogradskii-Hamilton principle), the Ostrogradskii-Euler equations, which are generalizations of the Euler-Lagrange equations, can be derived (for example, see Refs. [15, 16]). The equation (A.1) for the $\Phi(x)$-field can be obtained by means of the variation of the Lagrangian $L(x)$ with respect to the $\Phi(x)^{+}$-field and their derivatives. The equation for the $\Phi(x)^{+}$-field, similar to (A.1), can be derived by means of the variation of the $L(x)$ Lagrangian with respect to the $\Phi(x)$-field and their derivatives. In the terms of the definitions (16) the Ostrogradskii-Euler equation for the $\Phi(x)$ field may be written as

$$
\frac{\partial L}{\partial \Phi^{+}}+\sum_{n=1}^{N}(-1)^{n} \partial x_{\mu}{ }^{n} \cdot \frac{\partial L}{\partial \Phi x_{\mu}^{+n}}=0
$$

The total Lagrangian equals

$$
L(x)=L(x)_{\text {free }}+L(x)_{\text {int }} .
$$

The $L(x)_{\text {free }}$-part of Lagrangian allows one to derive the homogeneous equation (1) from the equation (18) and the $L(x)_{\text {int }}$-part leads to the right hand of the equation (A.1). The Lagrangian for homogeneous Klein-Gordon equation can be written as

$$
L(x)_{\text {free }}=-S_{N} \Phi^{+}(x) \Phi(x)-\sum_{n=1}^{N}(-1)^{n} S_{N-n} \Phi x_{\mu}^{+n} \Phi x_{\mu}^{n}
$$

The Lagrangian of an interaction is given by

$$
L(x)_{\mathrm{int}}=\Phi^{+}(x) \eta(x)+\Phi(x) \eta^{+}(x)
$$

A substitution of the (18)-(20) Lagrangians into the Ostrogradskii-Euler equation (17) gives the generalized KleinGordon equation (A.1) at arbitrary quantity of generations in a kind (or a family or a dynasty).

\section{Energy-momentum tensor for total fields}

The Lagrangian (19) for free total fields (4) does not depend on the $x$ coordinates explicitly. Therefore, we can expect that some conserved values (some first integrals of the equations) must exist. In the case of scalar field for one particle the conserved values constitute the energy-momentum tensor. In this case the Lagrangian includes the partial derivatives of the fields of first order. The Lagrangian (19) for the total fields (corresponding to the kind with $N$ generations) includes the partial derivatives of the $N$ order. The total derivatives of the Lagrangian (19) with respect to each coordinate equal

$$
\frac{d L}{d x_{\mu}}=\frac{\partial L}{\partial \Phi} \Phi_{\mu}+\sum_{n=1}^{N} \frac{\partial L}{\partial \Phi x_{\rho}^{n}} \cdot \partial_{\mu} \Phi x_{\rho}^{n}+\Phi \leftrightarrow \Phi^{+}
$$

After the substitution of the $\frac{\partial L}{\partial \Phi}$ term from the equation (17) the derivatives (21) are given by

$$
\frac{d L}{d x_{\mu}}=\Phi_{\mu} \cdot \sum_{n=1}^{N}(-1)^{n+1} \partial x_{\rho}^{n} \frac{\partial L}{\partial \Phi x_{\rho}^{n}}+\sum_{n=1}^{N} \frac{\partial L}{\partial \Phi x_{\rho}^{n}} \cdot \partial_{\mu} \Phi x_{\rho}^{n}+\Phi \leftrightarrow \Phi^{+}
$$

The right hand of (22) must be presented as a sum of total derivatives with respect to the coordinates. In particular, the right hand of (22) equal: 


$$
\begin{array}{ll}
\text { for } n=1 & \frac{d L}{d x_{\mu}}=\partial_{\nu}\left(\frac{\partial L}{\partial \Phi_{v}} \Phi_{\mu}\right)+\Phi \leftrightarrow \Phi^{+} \\
\text {for } n=2 & \frac{d L}{d x_{\mu}}=\partial_{\nu}\left[-\Phi_{\mu} \cdot \partial_{\rho}+\Phi_{\mu \rho}\right] \frac{\partial L}{\partial \Phi_{v \rho}}+\Phi \leftrightarrow \Phi^{+} \\
\text {for } n=3 & \frac{d L}{d x_{\mu}}=\partial_{\nu}\left[\Phi_{\mu} \cdot \partial_{\rho_{1}} \partial_{\rho_{2}}-\Phi_{\mu \rho_{1}} \partial_{\rho_{2}}+\Phi_{\mu \rho_{1} \rho_{2}}\right] \frac{\partial L}{\partial \Phi_{v \rho_{1} \rho_{2}}}+\Phi \leftrightarrow \Phi^{+}
\end{array}
$$

The use of total derivatives permits one to introduce the $E(x)_{\mu v}$ tensor, which is the generalization of the $T(x)_{\mu \nu}$ energy-momentum tensor for one particle (e.g., in Refs. [1-8])

$$
E(x)_{\mu \nu}=\sum_{n=1}^{N}(-1)^{n+1}\left\{\begin{array}{l}
{\left[\begin{array}{l}
\Phi_{\mu} \partial_{\rho_{1}} \partial_{\rho_{2}} \cdots \partial_{\rho_{n-1}}-\Phi_{\mu \rho_{1}} \partial_{\rho_{2}} \partial_{\rho_{3}} \cdots \partial_{\rho_{n-1}}+ \\
\Phi_{\mu \rho_{1} \rho_{2}} \partial_{\rho_{3}} \partial_{\rho_{4}} \cdots \partial_{\rho_{n-1}}-\cdots+(-1)^{n+1} \Phi_{\mu \rho_{1} \rho_{2} \cdots \rho_{n-1}}
\end{array}\right] \times} \\
\times \frac{\partial L}{\partial \Phi_{\nu \rho_{1} \rho_{2} \cdots \rho_{n-1}}}+\left[\Phi \leftrightarrow \Phi^{+}\right]
\end{array}\right\}-g_{\mu \nu} L .
$$

The $E(x)_{\mu v}$ tensor is symmetric with respect to the $\mu$ index and the $v$ index. The four-dimension divergence of the $E(x)_{\mu \nu}$ tensor vanishes similarly to the $T(x)_{\mu \nu}$ energy-momentum tensor. Therefore, the vector

$$
\bar{P}_{\mu}=\int E(x)_{\mu 0} d^{3} x
$$

must be conserved, i.e., components of this vector do not depend on the $x_{0}$ time. For quantized fields the components of this vector are operators. We shall consider matrix elements of these operators between one-particle states for the same particles.

At first, we calculate the contribution of the Lagrangian (19) to the $E(x)_{\mu v}$ tensor.

$$
\begin{aligned}
& -\int L(x) d^{3} x=\frac{1}{(2 \pi)^{3}} \sum_{k_{1}, k_{2}=1}^{N} \sqrt{A_{k_{1}}}\left(\sqrt{A_{k_{2}}}\right)^{*} \exp \left[i\left(\delta_{k_{1}}-\delta_{k_{2}}\right)\right] \times \\
& \times \int d^{3} x \int \frac{d^{3} p_{1} d^{3} p_{2}}{4 \omega_{k_{1}} \omega_{k_{2}}}\left\{\exp \left[i\left(-p_{1}+p_{2}\right) x\right] \cdot a\left(\vec{p}_{2}\right)_{k_{2}}^{+} a\left(\vec{p}_{1}\right)_{k_{1}}+b\left(\vec{p}_{1}\right)_{k_{1}} b\left(\vec{p}_{2}\right)_{k_{2}}^{+} \cdot \exp \left[i\left(p_{1}-p_{2}\right) x\right]\right\} \times \\
& \times\left[S_{N}+\sum_{n=1}^{N}\left(-\left(p_{1}, p_{2}\right)^{n} S_{N-n}\right)\right]= \\
& =\sum_{k=1}^{N}\left|A_{k}\right| \int \frac{d^{3} p}{4 \omega_{k}^{2}}\left[a(\vec{p})_{k}^{+} a(\vec{p})_{k}+b(\vec{p})_{k} b(\vec{p})_{k}^{+}\right] \cdot\left[S_{N}+\sum_{n=1}^{N}\left(-m_{k}{ }^{2}\right)^{n} S_{N-n}\right]= \\
& =\sum_{k=1}^{N}\left|A_{k}\right| \int \frac{d^{3} p}{4 \omega_{k}^{2}}\left[a(\vec{p})_{k}^{+} a(\vec{p})_{k}+b(\vec{p})_{k} b(\vec{p})_{k}^{+}\right] \cdot \prod_{n=1}^{N}\left(m_{n}{ }^{2}-m_{k}{ }^{2}\right)=0
\end{aligned}
$$

The integration in (26) with respect to the spatial coordinates gives the $(2 \pi)^{3} \delta\left(\vec{p}_{1}-\vec{p}_{2}\right)$-function. As the $\bar{P}_{\mu}$-vector does not depend on a time, the components of the $p_{1}$ momentum and the $p_{2}$ momentum must be equal $\left(p_{1}{ }^{2}=p_{2}{ }^{2}=m_{k}{ }^{2}\right)$. We assume that the quantity of generations is enough large for a convergence of the integrals in (26), similarly to the (9) relation. The $\prod_{n=1}^{N}\left(m_{n}{ }^{2}-m_{k}{ }^{2}\right)$-factor in (26) vanishes for arbitrary $k$ number generation.

The derivatives of the Lagrangian (19) can be written as 


$$
\frac{\partial L}{\partial \Phi x^{n}{ }_{\mu}}=(-1)^{n-1} S_{N-n} \Phi x^{+n}, \quad \frac{\partial L}{\partial \Phi x_{\mu}^{+n}}=(-1)^{n-1} S_{N-n} \Phi x^{n}{ }_{\mu}
$$

Now we find the vector of the four-momentum $\bar{P}_{\mu}$

$$
\begin{aligned}
& \bar{P}=(\bar{H}, \overrightarrow{\bar{P}})=\frac{1}{(2 \pi)^{3}} \sum_{k_{1}, k_{2}=1}^{N} \sqrt{A_{k_{1}}}\left(\sqrt{A_{k_{2}}}\right)^{*} \exp \left[i\left(\delta_{k_{1}}-\delta_{k_{2}}\right)\right] \times \\
& \times \int d^{3} x \int \frac{d^{3} p_{1} d^{3} p_{2}}{4 \omega_{k_{1}} \omega_{k_{2}}}\left\{\exp \left[i\left(-p_{1}+p_{2}\right) x\right] \cdot a\left(\vec{p}_{2}\right)_{k_{2}}{ }^{+} a\left(\vec{p}_{1}\right)_{k_{1}}+b\left(\vec{p}_{1}\right)_{k_{1}} b\left(\vec{p}_{2}\right)_{k_{2}}{ }^{+} \cdot \exp \left[i\left(p_{1}-p_{2}\right) x\right]\right\}
\end{aligned}
$$

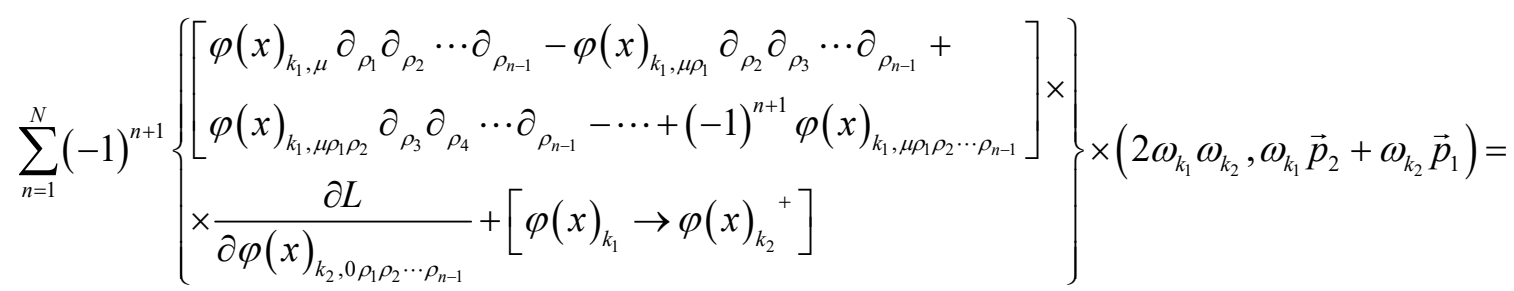

$$
\begin{aligned}
& =\sum_{k=1}^{N}\left|A_{k}\right| \int \frac{d^{3} p}{2 \omega_{k}}\left(\omega_{k}, \vec{p}\right) \cdot\left[a(\vec{p})_{k}^{+} a(\vec{p})_{k}+b(\vec{p})_{k} b(\vec{p})_{k}^{+}\right] \cdot Z\left(m_{k}^{2}\right) .
\end{aligned}
$$

Again the integration with respect to spatial coordinates in (28) leads to the $p_{1}=p_{2}, k_{1}=k_{2}$ equalities, similarly to (26). The $Z\left(m_{k}{ }^{2}\right)$ factors are determined by the derivatives of the fields $\varphi(x)_{k}(4)$ for the $k$ number generations:

$$
\begin{aligned}
& \varphi(x)_{k, \mu \mu \rho_{1} \rho_{2} \cdots \rho_{n-2}}=-m_{k}^{2} \varphi(x)_{k, \rho_{1} \rho_{2} \cdots \rho_{n-2}}, \\
& \varphi(x)^{+}{ }_{k, \mu \mu \rho_{1} \rho_{2} \cdots \rho_{n-2}}=-m_{k}^{2} \varphi(x)_{k, \rho_{1} \rho_{2} \cdots \rho_{n-2}}, \\
& \varphi(x)_{k, \mu \rho_{1} \rho_{2} \cdots \rho_{n-1}}^{+} \varphi(x)_{k, \mu v_{1} \nu_{2} \cdots v_{n-1}}=m_{k}{ }^{2} \cdot \varphi(x)^{+}{ }_{k, \rho_{1} \rho_{2} \cdots \rho_{n-1}} \varphi(x)_{k, v_{1} \nu_{2} \cdots v_{n-1}}, \\
& Z\left(m_{k}{ }^{2}\right)=\sum_{n=1}^{N}(-1)^{n-1} n \cdot\left(m_{k}{ }^{2}\right)^{n-1} \cdot S_{N-n} .
\end{aligned}
$$

Then we can write using (A.5)

$$
\begin{aligned}
& Z\left(q^{2}\right)=\sum_{n=1}^{N}(-1)^{n-1} n \cdot\left(q^{2}\right)^{n-1} \cdot S_{N-n}= \\
& (-1)^{N-1} \frac{d}{d q^{2}} \sum_{n=1}^{N}(-1)^{N-n}\left(q^{2}\right)^{n} \cdot S_{N-n}=(-1)^{N-1} \frac{d}{d q^{2}} \prod_{n=1}^{N}\left(q^{2}-m_{n}{ }^{2}\right), \\
& Z\left(m_{k}{ }^{2}\right)=\left.(-1)^{N-1} \frac{d}{d q^{2}} \prod_{n=1}^{N}\left(q^{2}-m_{n}{ }^{2}\right)\right|_{q^{2}=m_{k}{ }^{2}}=A_{k}^{-1}=(-1)^{k+1}\left|A_{k}{ }^{-1}\right| .
\end{aligned}
$$

After calculations of derivatives in (31) we must put $q^{2}=m_{k}{ }^{2}$. Now the energy-momentum vector $\bar{P}$ for the kind of particles with the $N$ generations is given by

$$
\bar{P}=(\bar{H}, \overrightarrow{\bar{P}})=\sum_{k=1}^{N}(-1)^{k+1} \int \frac{d^{3} p}{2 \omega_{k}}\left(\omega_{k}, \vec{p}\right) \cdot\left[a(\vec{p})_{k}^{+} a(\vec{p})_{k}+b(\vec{p})_{k} b(\vec{p})_{k}^{+}\right]
$$

This vector can be presented in terms of normal products of the operators similarly to the Hamiltonian for one particle (Refs. [1-8])

$$
\bar{P}_{\text {norm }}=\left(\bar{H}_{n o r m}, \overrightarrow{\bar{P}}_{n o r m}\right)=\sum_{k=1}^{N}(-1)^{k+1} \int \frac{d^{3} p}{2 \omega_{k}}\left(\omega_{k}, \vec{p}\right) \cdot\left[a(\vec{p})_{k}^{+} a(\vec{p})_{k}+b(\vec{p})_{k}^{+} b(\vec{p})_{k}\right] .
$$


The contribution to $\bar{H}_{n o r m}$ for $k=1$ in (33) equals the Hamiltonian of particle (Refs. [1-8]). The $\bar{H}_{\text {norm }}$ value in (33) can be named as the generalized Hamiltonian or the total Hamiltonian or the Hamiltonian of particle generations. The total Hamiltonian (33) includes the sign factors $(-1)^{k+1}$ in each term. Calculations of the eigenvalues for the total Hamiltonian with the use of the positive metrics give positive and negative values. Such calculations confirm known results that the particle energies are not positively determined for Lagrangians including higher derivatives of fields. Therefore, we consider the eigenvalues for the total Hamiltonian with the use of the indefinite metrics (5). Then for the particle with the $q$ momentum $\left(q=\left(\omega_{q k}, \vec{q}\right), \omega_{q k}=\sqrt{\vec{q}^{2}+m_{k}^{2}}\right)$ of the $k$ number generation the eigenvalues of the 4momentum may be derived

$$
\begin{aligned}
& \bar{P}_{n o r m, \mu}\left|q, k_{1}\right\rangle=\sum_{k=1}^{N}(-1)^{k+1} \int \frac{d^{3} p}{2 \omega_{p k}} \cdot p_{\mu}\left[a(\vec{p})_{k}^{+} a(\vec{p})_{k}+b(\vec{p})_{k}^{+} b(\vec{p})_{k}\right] a^{+}(\vec{q})_{k_{1}}|0\rangle= \\
& =\sum_{k=1}^{N}(-1)^{k+1} \int \frac{d^{3} p}{2 \omega_{p k}} \cdot p_{\mu} \cdot a(\vec{p})_{k}^{+} 2 \omega_{p k}(-1)^{k+1} \delta_{k k_{1}} \delta(\vec{p}-\vec{q})|0\rangle=q_{\mu}\left|q, k_{1}\right\rangle
\end{aligned}
$$

where $p=\left(\omega_{p k}, \vec{p}\right)$. The Eqs. (34) are valid for particles. But similar equations can be written for antiparticles also. Thus, the eigenvalues of the total Hamiltonian for all the particle generations are positive at the use of the indefinite metrics. This result is important. Therefore, it is of interest the consideration of different consequences of the indefinite metrics.

\section{Consequences of indefinite metrics}

The conserved $\bar{P}$ energy-momentum vector (32) has been derived from a condition that the Lagrangian (19) does not depend on $x$-coordinates explicitly. Now we calculate the vacuum average of the $\bar{H}$ - operator in (32)

$$
\begin{gathered}
\langle 0|\bar{H}| 0\rangle=\sum_{k=1}^{N}(-1)^{k+1} \int \frac{d^{3} p}{2}\left\langle 0\left|\left[a(\vec{p})_{k}^{+} a(\vec{p})_{k}+b(\vec{p})_{k} b(\vec{p})_{k}^{+}\right]\right| 0\right\rangle= \\
=\left\langle 0\left|\bar{H}_{n o r m}\right| 0\right\rangle+\sum_{k=1}^{N}(-1)^{k+1} \int \frac{d^{3} p}{2}\left\langle 0\left|\left[b(\vec{p})_{k}, b(\vec{p})_{k}^{+}\right]\right| 0\right\rangle=0+\sum_{k=1}^{N} \delta^{(3)}(\vec{o}) \int \omega_{k} d^{3} p=\infty \\
\langle 0|\bar{H}| 0\rangle=\sum_{k=1}^{N}(-1)^{k+1} \frac{d^{3} p}{2}\left\langle 0\left|\left[a(\vec{p})_{k}^{+} a(\vec{p})_{k}+b(\vec{p})_{k} b(\vec{p})_{k}^{+}\right]\right| 0\right\rangle= \\
=\left\langle 0\left|\bar{H}_{\text {norm }}\right| 0\right\rangle+\sum_{k=1}^{N}(-1)^{k+1} \int \frac{d^{3} p}{2}\left\langle 0\left|\left[b(\vec{p})_{k}, b(\vec{p})_{k}{ }^{+}\right]\right| 0\right\rangle=0+\sum_{k=1}^{N} \delta^{(3)}(\vec{o}) \int \omega_{k} d^{3} p=\infty
\end{gathered}
$$

Thus, the vacuum average (32) of the energy is infinite, similarly to such value for one particle (Refs [1-8]). Note that vacuum average of the $\bar{H}_{\text {norm }}$ total Hamiltonian (33) vanishes. As the $\delta^{(3)}(\vec{o})$-value equals the spatial volume, the density for the vacuum average (32) of the energy is infinite too.

Further the $\bar{P}_{\text {norm }}$ energy-momentum vector (33) will be considered without the norm -index (i.e., the operators in normal forms will be consider).

For arbitrary function of the $\bar{P}$-momentum operators we may write

$$
f(\bar{P})|q, k\rangle=f(q)|q, k\rangle \text {. }
$$

In particular, the equalities

$$
\exp ( \pm i \bar{P} x)|q, k\rangle=\exp ( \pm i q x)|q, k\rangle
$$

are valid at an use of the indefinite metrics.

Consider now the commutator of the total field and the operator of the total momentum (33) using the indefinite metrics: 


$$
\begin{aligned}
& {\left[\Phi(x), \bar{P}_{\mu}\right]=\left[\begin{array}{l}
\frac{1}{\sqrt{(2 \pi)^{3}}} \sum_{k=1}^{N} \sqrt{A_{k}} e^{i \delta_{k}} \int \frac{d^{3} p}{2 \omega_{k}}\left(e^{-i p x} a(\vec{p})_{k}+e^{i p x} b(\vec{p})_{k}^{+}\right), \\
\sum_{k_{1}=1}^{N}(-1)^{k_{1}+1} \int \frac{d^{3} q}{2 \omega_{q}} q_{\mu} \cdot\left(a(\vec{q})_{k_{1}}^{+} a(\vec{q})_{k_{1}}+b(\vec{q})_{k_{1}}^{+} b(\vec{q})_{k_{1}}\right)
\end{array}\right]=} \\
& =\frac{1}{\sqrt{(2 \pi)^{3}}} \sum_{k=1}^{N} \sqrt{A_{k}} e^{i \delta_{k}} \int \frac{d^{3} p}{2 \omega_{p k}} p_{\mu}(-1)^{k+1}(-1)^{k_{1}+1} \cdot 2 \omega_{p k} \cdot \delta_{k k_{1}} \cdot\left(e^{-i p x} a(\vec{p})_{k}-e^{i p x} b(\vec{p})_{k}^{+}\right)=i \frac{\partial \Phi(x)}{\partial x_{\mu}}
\end{aligned}
$$

The commutators (38) have the same forms as the commutators for one field and the Hamiltonian for one particle in Refs. [1-8]. Therefore, an use of the commutator (38) allows one to derive

$$
\Phi(x)=\exp (i \bar{P} x) \Phi(0) \exp (-i \bar{P} x)
$$

Using (36) and (39), matrix elements may be written as

$$
\left\langle q_{2}|\Phi(x)| q_{1}\right\rangle=\exp \left[i x\left(q_{2}-q_{1}\right)\right] \cdot\left\langle q_{2}|\Phi(0)| q_{1}\right\rangle
$$

The commutator of the particle annihilation operator and the total Hamiltonian (33) is given by

$$
\begin{aligned}
& {\left[a(\vec{q})_{k_{1}}, \bar{H}\right]=\sum_{k=1}^{N}(-1)^{k+1} \int \frac{d^{3} p}{2}\left[a(\vec{q})_{k_{1}}, a(\vec{p})_{k}^{+}\right] a(\vec{p})_{k}=} \\
& =\sum_{k=1}^{N}(-1)^{k+1} \int \frac{d^{3} p}{2}(-1)^{k_{1}+1} 2 \omega_{p k} \delta_{k k_{1}} \delta(\vec{p}-\vec{q}) a(\vec{p})_{k}=\omega_{q k_{1}} a(\vec{q})_{k_{1}} .
\end{aligned}
$$

Similarly, the commutators of the total Hamiltonian with the antiparticle annihilation operator and the creation operators can be derived:

$$
\left[a(\vec{q})_{k_{1}}^{+}, \bar{H}\right]=-\omega_{q k_{1}} a(\vec{q})_{k_{1}}^{+},\left[b(\vec{q})_{k_{1}}, \bar{H}\right]=\omega_{q k_{1}} b(\vec{q})_{k_{1}},\left[b(\vec{q})_{k_{1}}^{+}, \bar{H}\right]=-\omega_{q k_{1}} b(\vec{q})_{k_{1}}^{+} .
$$

If $\bar{H}\left|q_{1}, k_{1}\right\rangle=E\left(k_{1}\right)\left|q_{1}, k_{1}\right\rangle$ then we can write

$$
\begin{aligned}
& \bar{H} a\left(\vec{q}_{2}\right)_{k_{2}}\left|q_{1}, k_{1}\right\rangle=a\left(\vec{q}_{2}\right)_{k_{2}} \bar{H}\left|q_{1}, k_{1}\right\rangle-\left[a\left(\vec{q}_{2}\right)_{k_{2}}, \bar{H}\right]\left|q_{1}, k_{1}\right\rangle=\left(E\left(k_{1}\right)-\omega_{q_{2} k_{2}}\right) a\left(\vec{q}_{2}\right)_{k_{2}}\left|q_{1}, k_{1}\right\rangle, \\
& \bar{H} a\left(\vec{q}_{2}\right)_{k_{2}}^{+}\left|q_{1}, k_{1}\right\rangle=\left(E\left(k_{1}\right)+\omega_{q_{2} k_{2}}\right) a\left(\vec{q}_{2}\right)_{k_{2}}^{+}\left|q_{1}, k_{1}\right\rangle, \\
& \bar{H} b\left(\vec{q}_{2}\right)_{k_{2}}\left|q_{1}, k_{1}\right\rangle=\left(E\left(k_{1}\right)-\omega_{q_{2} k_{2}}\right) b\left(\vec{q}_{2}\right)_{k_{2}}\left|q_{1}, k_{1}\right\rangle, \\
& \bar{H} b\left(\vec{q}_{2}\right)_{k_{2}}^{+}\left|q_{1}, k_{1}\right\rangle=\left(E\left(k_{1}\right)+\omega_{q_{2} k_{2}}\right) b\left(\vec{q}_{2}\right)_{k_{2}}^{+}\left|q_{1}, k_{1}\right\rangle .
\end{aligned}
$$

From the relations (41-43) we may conclude that indeed the $a\left(\vec{q}_{2}\right)_{k_{2}}\left(b\left(\vec{q}_{2}\right)_{k_{2}}\right)$ operators are the annihilation operators of particle (antiparticle) with the $\vec{q}_{2}$-momentum from the $k_{2}$ generation number and $a\left(\vec{q}_{2}\right)_{k_{2}}{ }^{+}\left(b\left(\vec{q}_{2}\right)_{k_{2}}{ }^{+}\right)$ operators are similar creation operators of particle (antiparticle).

Consider the operators

$$
N_{a}(p, k)=\frac{(-1)^{k+1}}{2 \omega_{p k}} a(\vec{p})_{k}^{+} a(\vec{p})_{k}, \quad N_{b}(p, k)=\frac{(-1)^{k+1}}{2 \omega_{p k}} b(\vec{p})_{k}^{+} b(\vec{p})_{k},
$$

and states with $n$ particles and $l$ antiparticles $\left|q_{1}, k_{1} ; q_{2}, k_{2} ; \cdots ; q_{n}, k_{n} ; q_{n+1}, k_{n+1} ; \cdots ; q_{n+l}, k_{n+l}\right\rangle$. 
Then we can write:

$$
\begin{aligned}
& \int d^{3} p N_{a}(p, k)\left|q_{1}, k_{1} ; q_{2}, k_{2} ; \cdots ; q_{n}, k_{n} ; q_{n+1}, k_{n+1} ; \cdots ; q_{n+l}, k_{n+l}\right\rangle= \\
& =n\left|q_{1}, k_{1} ; q_{2}, k_{2} ; \cdots ; q_{n}, k_{n} ; q_{n+1}, k_{n+1} ; \cdots ; q_{n+l}, k_{n+l}\right\rangle \\
& \int d^{3} p N_{b}(p, k)\left|q_{1}, k_{1} ; q_{2}, k_{2} ; \cdots ; q_{n}, k_{n} ; q_{n+1}, k_{n+1} ; \cdots ; q_{n+l}, k_{n+l}\right\rangle= \\
& =l\left|q_{1}, k_{1} ; q_{2}, k_{2} ; \cdots ; q_{n}, k_{n} ; q_{n+1}, k_{n+1} ; \cdots ; q_{n+l}, k_{n+l}\right\rangle
\end{aligned}
$$

Thus, the $N_{a}(p, k)\left(N_{b}(p, k)\right)$ is an operator of a density for particle (antiparticle) quantity in a momentum space.

The use of the indefinite metrics permits one to derive the relations (36-43), (45) for total $\Phi(x), \Phi(x)^{+}$fields. These relations have the same forms as corresponding relations for one field (for example, in Refs. [1-8]).

\section{CONCLUSION}

In present paper it is shown that chronological products of the total free fields (4) for spinless particles are related to the Green functions (11) of generalized Klein-Gordon equations (A.1) at arbitrary $N$-number, which equals a quantity of particle generations in a kind $(N \geq 3)$. The Lagrangians (18-20) for spinless fields allow one to derive the generalized Klein-Gordon equations at arbitrary $N$-number as consequence of the least action principle. These Lagrangians have been used to obtain the energy-momentum vectors $(32,33)$. It is shown that positive eigenvalues of total Hamiltonians for all the particles in a kind can be derived using the indefinite metrics with the relations (5). In an addition, the equal-time commutators of total scalar fields [12], the relations between the chronological products of the total free fields and the Green functions (11) of generalized Klein-Gordon equations, and the relations (36-43), (45) can be just derived using the indefinite metrics. However, it is known that the indefinite metrics leads to negative probabilities for some states [17]. From the covariant normalization conditions (5) it is seen that the sign for particles with minimal masses (i.e., for $k=1$ ) is positive. Therefore, particles for $k=1$ may be stable. But all particles for even $k$ (i.e., for negative sign factors in (5), (32), (33), (44)) must be unstable. It may be assumed that the negative probabilities will not appear in final results for observables values. This assumption may be valid due to the independence of the relations (36-43), (45) on the sign $(-1)^{k+1}$-factor in (5).

It may be assumed that the relations (38), (39) allow one to derive the $S$-matrix in terms of the interaction Lagrangian (20) including the total fields:

$$
S=T\left(\exp \left(i \int L(x)_{\mathrm{int}} d^{4} x\right)\right)
$$

In addition to the investigations for spinless total fields executed in present paper, it is of importance similar studies of spinor total fields. In this case the Lagrangians [13] for spinor total fields at arbitrary number of particle generations can be used. It is of interest the investigations of consequences of the Lagrangians (18)-(20) and the Lagrangians from Ref. [13] for electromagnetic interactions. These Lagrangians include higher derivatives of total fields. As it is known, the electromagnetic interactions with the charge of one particle can be derived by means of a change of partial derivatives on the 4-vector of electromagnetic potential (Refs [1-8]). Therefore, we can assume that for total fields the electromagnetic interactions with the electric charges can be derived by means of similar changes. At such changes the 4-vector of potential for the photonic kind must appear. The total field of the photonic kind is a sum of the fields for neutral vector massless particle (photon) and massive particles, by analogy with (A.2), (4). As the Lagrangians for generalized Klein-Gordon (Dirac) equations include higher derivatives, vertex functions for interactions of spinless (1/2-spin) particles with some number of photons can appear. These interactions correspond to a point in Feynman diagram (the contact interaction).

Acknowledgements

Author thanks V.I. Khrabustovsrii, S.D. Bronza, O.A. Osmaev, A.A. Zheltukhin for useful discussions.

\section{Generalized Klein-Gordon equations}

Appendix

The generalization of the Klein-Gordon equation proposed in Refs. [9, 10] is given by

$$
\left(\square+m_{1}^{2}\right)\left(\square+m_{2}^{2}\right) \ldots \ldots . .\left(\square+m_{N}^{2}\right) \Phi(x)=\eta(x),
$$


where $\Phi(x)$ is the field and $\eta(x)$ is the current (the field source). In momentum space the differential operator in (A.1) is the polynomial of the $N$ - degree. We consider the case of the polynomial with real non-negative different zeros at $m_{1}{ }^{2}<m_{2}{ }^{2}<m_{3}^{2}<\ldots .<m_{N}{ }^{2}$.

The general classical solution $\Phi_{c l}(x)$ of the linear equation (A.1) is the sum of the general solution of the corresponding homogeneous equation $\Phi(x)_{\text {free }}$ and partial solution $\Phi(x)_{n h}$ of non-homogeneous equation:

$$
\begin{gathered}
\Phi(x)_{\text {free }}=\int d^{4} q \sum_{k=1}^{N_{b}} \delta\left(q^{2}-m_{k}^{2}\right)\left[c_{k} e^{-i q x}+\tilde{c}_{k} e^{i q x}\right] \\
\Phi(x)_{n h}=\int \bar{G}(x-y) \eta(y) d^{4} y,
\end{gathered}
$$

where $c_{k}$ and $\tilde{c}_{k}$ are arbitrary constants. Thus, $\Phi(x)_{\text {free }}$ is the sum of the terms corresponding to particles with the same charges, parities, spin, but with different masses. Each term in (A.2) corresponding to number $k$ is the solution of the homogeneous Klein-Gordon equation as $\left(\square+m_{k}^{2}\right)\left(c_{k} e^{-i q x}+\tilde{c}_{k} e^{-i q x}\right) \delta\left(q^{2}-m_{k}^{2}\right)=0$. In Ref. [9] it is shown that the case of equal masses in Eq. (A.1) must be excluded. It was shown that the functions $\Phi(x)_{\text {free }}$ can include nonnormalizable terms if at least two masses are equal. Thus, the masses in the generalized Klein Gordon equation must be different. The $N$-number equals to the quantity of generations for spinless bosons and order of the equation (A.1) equals $2 N$.

The Green functions for the generalized Klein-Gordon equations (A.1) are given by

$$
\bar{G}(x)=\frac{1}{(2 \pi)^{4}} \int \frac{e^{-i q x} d^{4} q}{\left(-q^{2}+m_{1}^{2}\right)\left(-q^{2}+m_{2}^{2}\right) \ldots\left(-q^{2}+m_{N}^{2}\right)}=\frac{1}{(2 \pi)^{4}} \int \frac{e^{-i q x} d^{4} q}{P_{N}\left(q^{2}\right)},
$$

where $P_{N}\left(q^{2}\right)$ is the polynomial of the $N$ degree with respect to $q^{2}$. It is clear that the integrals in (A.4) can converge at $N \geq 3$, i.e., when the order of the equation (A.1) is greater than or equals six. Consequently for each spinless particle two (or greater) particles with the same charges, isospin, $C$ - and $P$ parity, but different masses, must exist in addition. We may say that such particles are members of some set (a family or a kind or a dynasty). In Eq. (A.2) $k$ is the number of the particle generation. We may assume that the quantity of members in kinds for the elementary particle is less than the quantity of member in kinds for the composite particle. Each particle belongs to some kind and some generation.

According to Refs. $[9,10]$, the rational fraction in (A.4) can be written as

$$
\begin{aligned}
& \frac{1}{P_{N}\left(q^{2}\right)}=\frac{1}{\left(-q^{2}+m_{1}^{2}\right)\left(-q^{2}+m_{2}^{2}\right) \ldots\left(-q^{2}+m_{N}^{2}\right)}=\sum_{k=1}^{N} \frac{A_{k}}{-q^{2}+m_{k}^{2}}, \\
& A_{k}=-\frac{1}{P_{N}^{\prime}\left(m_{k}^{2}\right)}=\lim _{q^{2} \rightarrow m_{k}^{2}} \frac{-q^{2}+m_{k}^{2}}{P_{N}\left(q^{2}\right)}, \quad A_{k}=(-1)^{k+1}\left|A_{k}\right| \\
& A_{k}^{-1}=\left.(-1)^{N-1} \frac{d}{d q^{2}} \prod_{n=1}^{N}\left(q^{2}-m_{n}^{2}\right)\right|_{q^{2}=m_{k}^{2}}
\end{aligned}
$$

The $A_{k}$ coefficients obey the relations:

$$
\begin{gathered}
\sum_{k=1}^{N} A_{k} m_{k}^{2 l}=0, \quad l=0,1,2, \ldots, N-2 \\
\sum_{k=1}^{N} A_{k} m_{k}^{2 N-2}=(-1)^{N+1}
\end{gathered}
$$

Using the equality (A.5) we may write the Green functions (A.4) of Eq. (A.1) in the form 


$$
\bar{G}(x)=\sum_{k=1}^{N} A_{k} D\left(x, m_{k}\right)
$$

where $D(x, m)$ is the Green function of the Klein-Gordon equation:

$$
D(x, m)=\frac{1}{(2 \pi)^{4}} \int \frac{e^{-i q x} d^{4} q}{-q^{2}+m^{2}}
$$

The causal Green functions (4) of generalized Klein-Gordon equation (A.1) may be written as

$$
\bar{G}(x)_{c}=\frac{1}{(2 \pi)^{4}} \int \frac{e^{-i q x} d^{4} q}{\left(-q^{2}+m_{1}^{2}-i \varepsilon\right)\left(-q^{2}+m_{2}^{2}-i \varepsilon\right) \ldots\left(-q^{2}+m_{N}^{2}-i \varepsilon\right)},
$$

where $\varepsilon$ is an infinitesimal positive number. The (A.5), (A.6), and (A.9) expressions have been used for calculation of the Green function (A.10). According to Ref. [1] the causal Green function (A.10) is expressed through the cylindrical $K_{1}\left(m \sqrt{x^{2}}\right)$ function, which has singularities on the light cone $\left(x^{2}=0\right)$. The series for the $K_{1}\left(m \sqrt{x^{2}}\right)$ function has been used from Refs. $[18,19]$. The use of the relations (A.6) at $l=0,1$ has allowed one to eliminate all the singularities. It has been shown that the integral (A.10) converges at $N \geq 3$ in all the space-time [20]. Thus, it may be concluded that minimal number of the generations in the kinds of the spinless particles equals three.

\section{REFERENCES}

1. Bogolubov N.N., Shirkov D.V. Introduction to theory of quantized fields. - Moscow: Nauka, 1967. - P. 465 (in Russian)

2. Schweber S.S. An introduction to relativistic quantum field theory. - Brandeis Univ. - Row, Peterson and Co. Evanston, Ill., N.Y., 1961; Izlftel'stvo Inostrannoj Literatury. - Moscow, 1963. - 843p. (in Russian)

3. Bjorken J.D., Drell S.D. Relativistic quantum fields. Relativistic quantized fields. Vol. 2. - N.Y.: Mc Graw-Hill Book Company, 1965; Moscow: Nauka, 1978. - 408p. (in Russian)

4. Itzykson C, Zuber J.-B. Quantum field theory. Vol. 1. - N.Y.: Mc Graw-Hill Book Company; Moscow: Mir, 1984. - 448p. (in Russion)

5. Barton G. Introduction to dispersion techniques in field theory. - New York, Amsterdam: Univ. of Sussex. W.A. Benjamin. Inc., 1965; Moscow: Atomizdat, 1968. - 392p. (in Russian)

6. Gaziorowicz S. Elementary particle physics. - New York-London-Sydney: John Wilej \& Sons Inc.; Moscow: Nauka, 1969. 743 p. (in Russian)

7. Akhiezer A.I., Peletminskii S.V. Fields and fundamental interactions. - Kiev: Naukova Dumka, 1986. - 552p. (in Russian)

8. Akhiezer A.I., Peletminskii S.V. Theory of fundamental interactions. - Kiev: Naukova Dumka, 1993. - 570p. (in Russian)

9. Kulish Yu. V., Rybachuk E.V. Divergences of integrals for Green functions and necessary existence of particle generations // Journal of Kharkiv National Univ. - 2011. - No.955. - Iss. 2(50). - P.4-14.

10. Kulish Yu., Rybachuk E.V. Necessary generalization of Klein-Gordon and Dirac equations and existence of particle generations // Problems of Atomic Science and Technology. - 2012. - No.1 (77). - P. 16-20.

11. Mathews J, Walker R.L. Mathematical methods of physics. - Calif. Inst. of Tech. - NewYork-Amsterdam: W.A. Benjamin Inc, 1964; Moscow: Anomizdat, 1972. - 400p. (in Russian)

12. Kulish Yu. V., Rybachuk E.V. Locality of quantized scalar fields for generations of particles // EEJP. - 2017. - Vol. 4. - No. 4. - P. 4-11.

13. Kulish Yu.V. Classification of particles at arbitrary quantity of generations. I. Hadrons // EEJP. - 2016. - Vol. 3. - No. 4. P. 22-33.

14. Korn G.A., Korn T.M. Mathematical handbook for scientists and engineers. Definitions, theorems and formulas for reference and review. - New York, San Francisco, Toronto, London, Sydney: Mc Graw Book Company, 1968; Moscow: Nauka, 1978. P. 34. (in Russian)

15. Esl'sgol'ts L.E. Variational calculus. - Moscow: Gostechizdat, 1952. (in Russian)

16. Myshkis A.D. Mathematics. Special courses for higher technical schools. - Moscow: Nauka, 1971. - 632p. (in Russian)

17. Nagy K.L. State vector spaces with indefinite metric in quantum field theory. - Budapest: Akademial Kiado, 1966; State vector spaces with indefinite metric in quantum field theory. Library of "Mathematics" Collection. - Moscow: Mir, 1969. - 136p. (in Russian)

18. Whittaker E.T., Watson G.N. A course of modern analysis. Vol. 2. - Cambridge: University Press, 1927; Moscow: Gosudarstvennoe izdatel'stvo fisiko-matematicheskoj literatuty, 1963. - P.215.

19. Berezhnoj Yu.A., Gakh A.G. Functions of theoretical physics. - V.N. Karazin Kharkov National University. - Kharkov. 2011. - 124p. (in Russian)

20. Kulish Yu.V. Elimination of singularities in causal Green functions for generalized Klein-Gordon and Dirac equations on light cone // EEJP. - 2016. - Vol. 3. - No. 3. - P.73-83. 